\title{
Molecular imaging of tumor angiogenesis using $\alpha \mathrm{v} \beta 3$-integrin targeted multimodal quantum dots
}

\author{
Willem J. M. Mulder · Karolien Castermans · Judy R. van Beijnum • \\ Mirjam G. A. oude Egbrink - Patrick T. K. Chin - Zahi A. Fayad • \\ Clemens W. G. M. Löwik · Eric L. Kaijzel · Ivo Que · Gert Storm • \\ Gustav J. Strijkers · Arjan W. Griffioen · Klaas Nicolay
}

Received: 8 January 2008/Accepted: 18 November 2008/Published online: 9 December 2008

(C) The Author(s) 2008. This article is published with open access at Springerlink.com

\begin{abstract}
Molecular imaging of angiogenesis is urgently needed for diagnostic purposes such as early detection, monitoring of (angiostatic) therapy and individualized therapy. Multimodality molecular imaging is a promising and refined technique to study tumor angiogenesis, which has so far been largely unexplored due to the lack of suitable multimodal contrast agents. Here, we report on the application of a novel $\alpha \mathrm{v} \beta 3$-specific quantum dot-based nanoparticle, which has been optimized for both optical and magnetic resonance detection of tumor angiogenesis. Upon intravenous injection of RGD-pQDs in tumor-bearing mice, intravital microscopy allowed the detection of angiogenically activated endothelium at cellular resolution with a small scanning window and limited penetration depth, while magnetic resonance imaging was used to
\end{abstract}

W. J. M. Mulder $(\bowtie) \cdot$ G. J. Strijkers · K. Nicolay

Biomedical NMR, Department of Biomedical Engineering,

Eindhoven University of Technology, P.O. Box 513, 5600 MB

Eindhoven, The Netherlands

e-mail: willem.mulder@mountsinai.org

W. J. M. Mulder · Z. A. Fayad

Department of Radiology, Translational and Molecular Imaging Institute and Imaging Science Laboratories, Mount Sinai School of Medicine, One Gustave L. Levy Place Box 1234, New York, NY 10029, USA

K. Castermans · J. R. van Beijnum · A. W. Griffioen Department of Pathology/Internal Medicine, Angiogenesis Laboratory, Research Institute for Growth and Development, Maastricht University \& University Hospital, P.O. Box 5800, 6202 AZ Maastricht, The Netherlands

M. G. A. oude Egbrink

Department of Physiology, Cardiovascular Research Institute

Maastricht, Maastricht University, Maastricht, The Netherlands visualize angiogenesis at anatomical resolution throughout the entire tumor. Fluorescence imaging allowed wholebody investigation of angiogenic activity. Using these quantum dots and the aforementioned imaging modalities, the angiogenic tumor vasculature was readily detected with the highest angiogenic activity occurring in the periphery of the tumor. This nanoparticle may be employed for multimodality imaging of a variety of diseases that are accompanied by activation of endothelial cells. Furthermore, the current technology might be developed for molecular imaging of other pathophysiological processes.

Keywords Quantum dots · Multimodality imaging · Angiogenesis - Magnetic resonance imaging · Intravital microscopy $\cdot$ Fluorescence imaging

\section{P. T. K. Chin}

Laboratory of Macromolecular and Organic Chemistry, Eindhoven University of Technology, P.O. Box 513, 5600 MB Eindhoven, The Netherlands

\section{W. G. M. Löwik · E. L. Kaijzel · I. Que}

Department of Endocrinology and Metabolic Diseases (C4-R), Leiden University Medical Centre, Albinusdreef 2, 2300 RC

Leiden, The Netherlands

\section{G. Storm}

Department of Pharmaceutics, Utrecht Institute for Pharmaceutical Sciences (UIPS), 3508 TB Utrecht, The Netherland 


\section{Introduction}

In vivo imaging methods to depict and characterize angiogenesis are becoming increasingly important for studying this process in both clinical and research settings [1]. The level of focus ranges from identifying sites of angiogenesis, via the quantification of the level of new blood vessel formation, to the biochemistry that lies at the basis of the angiogenic process [1]. In particular, tumor angiogenesis is an important target for in vivo imaging methods, both for diagnostic purposes and for the evaluation of novel anti-cancer treatment strategies based on the inhibition of angiogenesis [2]. The mechanism and efficacy of an applied therapy need to be evaluated accurately, preferably using a non-invasive technology that can be used in a longitudinal study design. Recently, a lot of effort has been put in the development of noninvasive molecular imaging approaches, aimed at visualizing the molecular and cellular processes that are related to angiogenesis [3]. A well-known marker of angiogenic blood vessels is the $\alpha \mathrm{v} \beta 3$-integrin [2, 4-7]. This integrin is strongly expressed on activated tumor endothelial cells [2], whereas it is only weakly expressed on resting endothelial cells of blood vessels in non-diseased tissue.

Three powerful in vivo molecular imaging methods for the investigation of angiogenesis are intravital microscopy (IVM), magnetic resonance imaging (MRI), and fluorescence imaging [8, 9]. The techniques are highly complementary. Intravital microscopy is able to probe tissues at the (sub)cellular level, be it with a small scanning window and often limited to structures exposed at the surface of cells, while MRI is capable of generating images of opaque tissue with a large scanning window at resolutions in the order of 50-100 $\mu \mathrm{m}$. Fluorescence imaging allows the investigation of whole small animals with high sensitivity and short acquisition times. The strong points of these techniques can be combined by the use of a single molecular imaging contrast agent that is optimized for parallel detection by both optical techniques, such as IVM and optical imaging, and MRI.

Over the last few years, quantum dots (semiconductor nanocrystalloid particles) have gained much interest for biological imaging purposes [10], because of their bright fluorescence, their excellent photo-stability, and their narrow and tunable emission spectrum [11-13]. We recently developed a novel quantum dot-based nanoparticle that is coated with a paramagnetic micellar shell to enable their detection with fluorescence imaging and MRI [14]. In the current study, we show the ability to utilize this nanoparticle to visualize ongoing tumor angiogenesis in tumorbearing mice using different imaging modalities.

\section{Methods}

Contrast agent

RGD-conjugated quantum dots with a paramagnetic coating (RGD-pQDs) were synthesized as described previously [14]. In short, high quality core shell $\mathrm{CdSe} / \mathrm{ZnS}$ core/shell QDs were synthesized by injection of precursors (Cd(acetate) 2 dissolved trioctylphosphine (TOP) and TOP-Se) into a hot coordinating solvent mixture (trioctylphosphine oxide/ hexadecylamine [TOPO/HDA]). This was followed by the synthesis of the zinc sulfide shell in a coordinating surfactant mixture of HDA/TOPO/stearic acid using zinc stearate and elemental sulfur in TOP as the precursors. A micellar and paramagnetic coating, comprised PEG-DSPE (1,2-distearoyl-sn-glycero-3-phosphoethanolamine- $N$-[methoxy(polyethylene glycol)-2000]), Mal-PEG-DSPE (1,2-distearoylsn-glycero-3-phosphoethanolamine- $N$-[maleimide(polyethylene glycol)2000]), and Gd-DTPA-BSA (Gd-DTPA-bis (stearylamide)) in a $0.4 / 0.1 / 0.5$ molar ratio was applied to the QDs. To that end, a 50-fold excess of the theoretical maximum lipid coverage was applied to the quantum dots by slowly dripping a QD/lipid mixture in chloroform to a $65^{\circ} \mathrm{C}$ HEPES buffer. The micelle-coated QDs were separated form the empty micelles by centrifugation. Approximately 50 cyclic RGD peptides [15] were conjugated to the lipidcoated quantum dots by sulfhydryl-maleimide coupling.

\section{Chorioallantoic membrane experiments}

Fertilized eggs of Lohman-selected White Leghorns were incubated for 3 days, as previously described [16], at $37^{\circ} \mathrm{C}$, $55 \%$ relative air humidity, while being rotated every hour. On day 3 , a rectangular window $\left(1 \times 1.5 \mathrm{~cm}^{2}\right)$ was made in the shell. Albumin $(2 \mathrm{ml})$ was withdrawn through the blunt end of the egg. The window was covered with transparent tape to prevent dehydration, and the eggs were further incubated without rotation until day 10. For analysis of tumor-induced angiogenesis in the chorioallantoic membrane (CAM) model, LS147T colon tumor cells were grown for 7 days onto the 10-day-old CAM. Immediately before the transplantation, the CAM was slightly damaged by removing its upper peridermal layer by quick adhesion and removal of lens tissue paper. On day 17, RGD-pQDs were injected intravenously under microscopic guidance and were allowed to circulate for $1 \mathrm{~h}$. Tumor blood vessels and tumor tissue were excised and placed in Petri dishes. Fluorescence microscopy and brightfield images of the tissue were taken with an inverted microscope equipped with a Nikon F301 camera. 
Mouse tumor model

On day 0, thirteen 9-week-old male C57B1/6 mice (Charles River, Maastricht, The Netherlands) were inoculated with $10^{5}$ B16F10 mouse melanoma cells subcutaneously on the right flank. Between day 14 and 19, when tumors had grown to a size of $500-1,000 \mathrm{~mm}^{3}$, mice were used for IVM and MRI measurements.

All experiments were approved by the Institutional Ethical Review Committee for animal experiments of the Maastricht University.

\section{Intravital microscopy}

Intravital microscopy experiments were performed on a Leitz intravital microscope, equipped with a salt water immersion objective (SW25, numerical aperture 0.6), a $100 \mathrm{~W}$ mercury lamp, a Leitz Ploemopak incident illuminator (with appropriate filter sets) and a fast, high resolution, low light performance digital camera (C9100-02 EM-CCD, Hamamatsu). Mice $(n=6)$ were anesthetized by subcutaneous administration of a mixture of ketamine $(0.1 \mathrm{mg} / \mathrm{g}$ b.w. Nimatek) and xylazine ( $0.02 \mathrm{mg} / \mathrm{g}$ b.w Sedamun; AUV, Cuijk, the Netherlands). Either $\alpha \mathrm{v} \beta 3$-specific RGD-pQDs or non-specific $\mathrm{pQDs}$ were injected through a catheter in the tail vein. The tumor and surrounding tissue were prepared free by making an incision in the hind limb and removing the skin. Body temperature was kept at $37^{\circ} \mathrm{C}$ by an infrared heating lamp. Up to $45 \mathrm{~min}$ after contrast agent injection, brightfield and fluorescence images of vessels in the tumor, in surrounding tissue, and in the ear of the animals were recorded on a hard disk. After the IVM experiments, mice were killed and the tumor, muscle tissue, spleen, kidney, and liver were frozen in isopentane for fluorescence microscopy.

\section{MRI}

MRI experiments were performed on a 6.3 Tesla horizontal bore magnet (Oxford Instruments, England) interfaced to a Bruker (Bruker, Ettlingen, Germany) MR Imaging console.

Mice $(n=4)$ were anesthetized with isoflurane $(1.5-2 \%$ in medical air) and placed in a home-built cradle, equipped with a mask for anesthesia gas supply and a warm water pad. An infusion line with contrast agent was placed and fixed in the tail vein in order to inject the contrast agent during the MRI experiment. The injection dose was $100 \mu \mathrm{l}$ of a RGD-pQDs suspension per animal, which corresponded with $0.5 \mathrm{mg}$ QDs. The cradle and the coil were placed in the MR scanner. Respiration was monitored with a balloon sensor connected to an ECG/respiratory unit (Rapid Biomedical, Würzburg, Germany).

First, multislice $\mathrm{T} 2$-weighted $(\mathrm{TR}=2,000 \mathrm{~ms}, \mathrm{TE}=$ $30 \mathrm{~ms}$, matrix size $=128 \times 128$, slice thickness $=1 \mathrm{~mm}$,
$\mathrm{NEX}=4, \mathrm{FOV}=3 \times 3 \mathrm{~cm}^{2}$ ) images were acquired to localize the tumor. Next, T1-weighted images were collected with a repetition time $\mathrm{TR}=800 \mathrm{~ms}$ and an echo time $\mathrm{TE}=9 \mathrm{~ms} \quad($ matrix size $=128 \times 128, \quad$ slice thickness $=1 \mathrm{~mm}, \mathrm{NEX}=16, \mathrm{FOV}=3 \times 3 \mathrm{~cm}^{2}$ ) and, from 21 consecutive slices, were generated before the contrast agent was injected. Images with the same parameters as described above were generated 45 min after injecting the contrast agent. The MRI data analysis was performed using Mathematica 5.0 (Wolfram Research Inc.).

The tumors were segmented by manually drawing regions of interest (ROIs) around the tumor area for all slices in the T2-weighted images. After the injection of the contrast agent, the pixel intensities in the tumor ROIs were compared to the pre-contrast intensities. A pixel was considered significantly enhanced when its intensity was increased by at least three times the mean noise level.

\section{Whole-animal fluorescence imaging}

Luciferase (luc)-expressing human renal carcinoma cells (RC21-luc) were injected (100,000 cells per $10 \mu \mathrm{l}$ PBS) in the renal capsule of Balb/c nude mice $(n=3)$, which led to the growth of luc-expressing carcinoma in the kidney. Ten weeks after tumor inoculation, luciferase expression was assessed $5 \mathrm{~min}$ after intraperitoneal (i.p.) injection of luciferin $(2 \mathrm{mg}$ in $30 \mu \mathrm{l}$ PBS). Total photon emission was acquired for $30 \mathrm{~s}$. Fluorescence imaging of both RGDpQDs and luciferase in vivo was accomplished using a peltier cooled CCD camera system NightOWL "UltraSense Frontlit" LB 981 (Berthold Technologies, Germany). For fluorescent imaging, a $525 \pm 25 \mathrm{~nm}$ wavelength filter (Omega Optical, Glen Spectra Limited, England) was attached to the CCD camera. A $470 \pm 10 \mathrm{~nm}$ wavelength filter (Omega Optical, Glen Spectra Limited, England) was inserted in front of the light source. Mice were anesthetized with isoflurane/oxygen placed in the imaging cabinet, and images were acquired $10 \mathrm{~min}$ after intracardial injection of RGD-pQDs at a camera exposure time of 1, 5, and $10 \mathrm{~s}$. Fluorescent and luminescent signals were expressed in photons $/ \mathrm{cm}^{2} / \mathrm{s}$ by using the WinLight 32 software (Berthold Technologies). The ROIs of the fluorescent and luminescent signals from the images were calculated by drawing a circle around the signals. In addition, color-enhanced overlays of luminescent and fluorescent images on photographic images were created using the WinLight software.

\section{Results}

LS174T melanoma tumor cells were grown on the CAM [16] for 7 days. RGD-pQDs, schematically depicted in Fig. 1a, were administered intravenously in a CAM vessel 
A

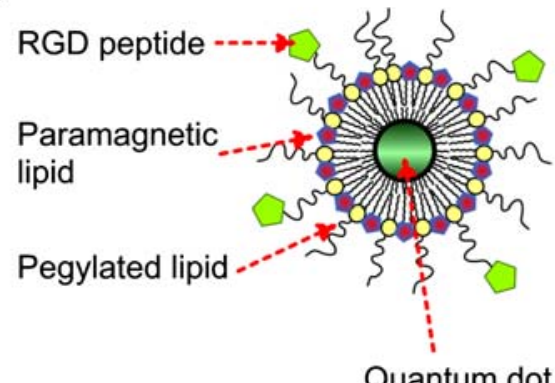

Fig. 1 Paramagnetic quantum dots target angiogenic vessels in the CAM model. a The nanoparticulate contrast agent consists of a quantum dot core covered by a micellar shell composed of pegylated phospholipid (PEG-DSPE) and Gd-DTPA-based lipids (Gd-DTPABSA), of which the latter have paramagnetic properties for MRI. Pentapeptidic peptides with the RGD-sequence in a cyclic confirmation are conjugated to the nanoparticle to provide specificity for the

under microscopic guidance and after $1 \mathrm{~h}$ the tumor tissue with the corresponding angiogenic blood vessels was excised and put in a Petri dish containing PBS. Fluorescence microscopy revealed a massive association of the contrast material with the angiogenic blood vessels only. In Fig. 1b and d, two typical fluorescence images are depicted. The corresponding bright field images are shown in
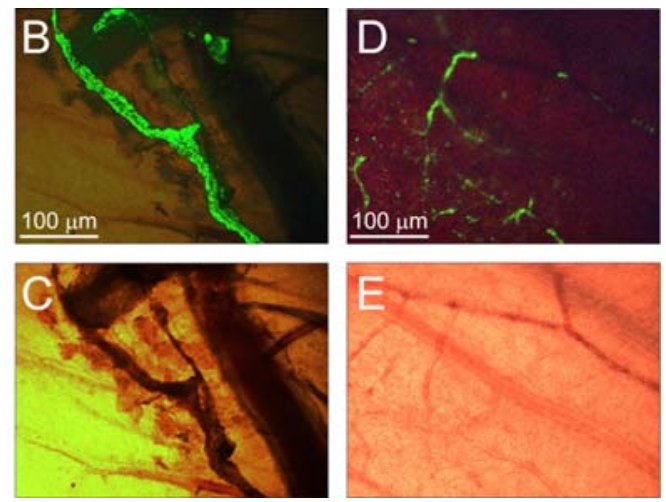

$\alpha \mathrm{v} \beta 3$-integrin, an angiogenesis-specific endothelial cell surface receptor. b Fluorescence images $(\mathbf{b}, \mathbf{d})$ and corresponding bright field images $(\mathbf{c}, \mathbf{e})$ of excised 17-day-old CAMs with topically growing LS174T human colon carcinoma tissue. The embryos of the fertilized eggs were intravenously injected with RGD-pQDs and the contrast agent was left to circulate for $1 \mathrm{~h}$

Fig. 1c and d, respectively. No association of the RGDQDs was found with mature blood vessels or vessels outside the tumor.

To demonstrate molecular imaging of tumor angiogenesis using RGD-pQDs at the cellular and macroscopic level, we used mice that were inoculated with B16F10 melanoma cells and had a tumor grown to a size of
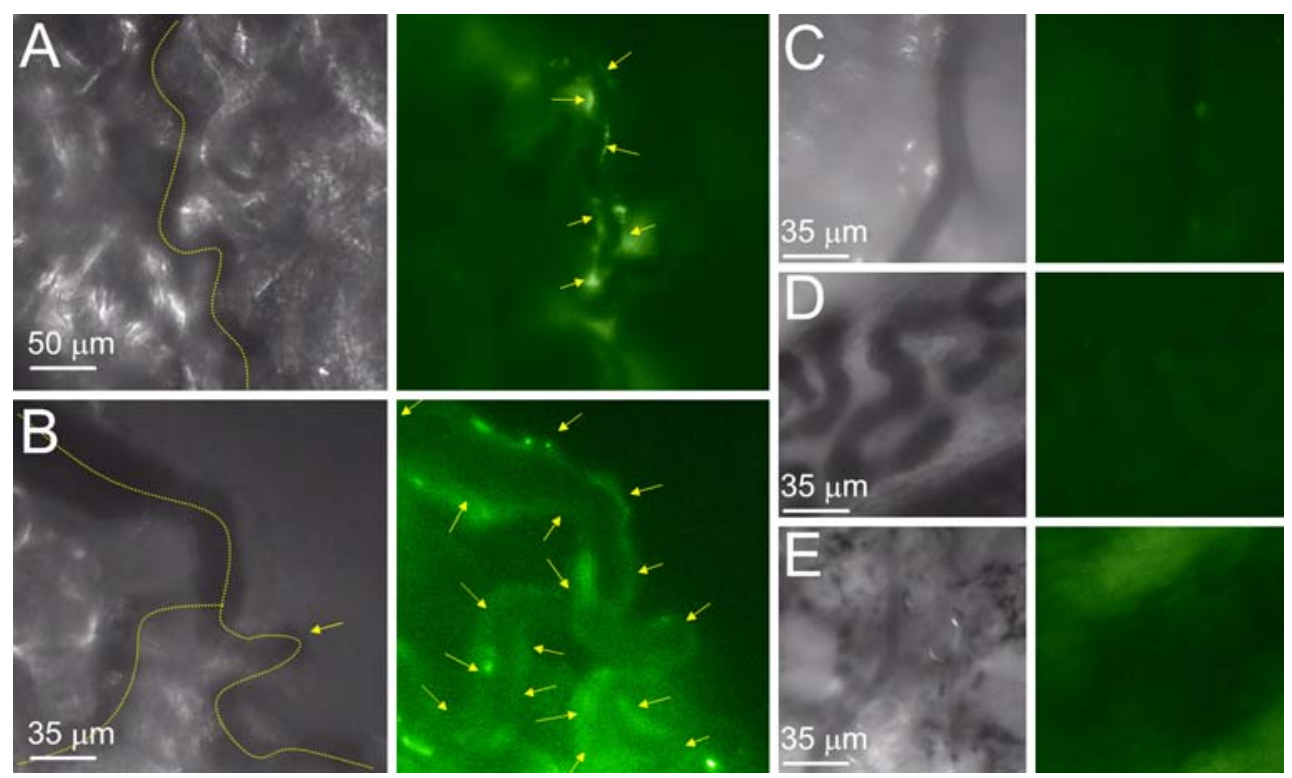

Fig. 2 RGD-labeled paramagnetic quantum dots (RGD-pQDs) specifically target tumor blood vessels in vivo IVM of microvessels in tumor-bearing mice (C57B16) after intravenous injection of RGDpQDs (a-c) and pQDs (d,e). Brightfield microscopy (left panels) was used to select blood vessels (indicated by the yellow lines). Fluorescence microscopy (right panels) revealed labeling of endothelial cells in tumor blood vessels of mice that were injected with
RGD-pQDs, as indicated by the yellow arrows (a,b). Endothelial cells in blood vessels of normal tissues (skin of the ear) in the same mouse showed no accumulation of contrast agent $(\mathbf{c})$. An animal that was injected with bare pQDs did not show association of contrast agent with the endothelial cells in tumor (d) or normal tissue blood vessels in the ear (e) 
$\sim 500 \mathrm{~mm}^{3}$. The animals were intravenously injected with the contrast agent via an infusion line in the tail vein and studied with either IVM or MRI in vivo.

Intravital microscopy was performed on anesthetized animals $(n=6)$, after removal of the skin to expose the tumor that was grown on the flank. Brightfield microscopy was applied to select a tumor region with angiogenic blood vessels showing their typical tortuous morphology. Thereafter, the RGD-pQDs were injected intravenously and IVM was performed continuously. At several time points post injection, fluorescence microscopy images were recorded at different sites in and around the tumor. Within 5-10 min, the RGD-pQDs attached to and labeled the endothelial cells in the tumor blood vessels. After $30 \mathrm{~min}$, the non-bound contrast agent was entirely cleared from the circulation. In Fig. 2a and b, typical brightfield images and corresponding fluorescence images of blood vessels in two different tumors are depicted, which were collected $30 \mathrm{~min}$ after the injection of RGD-pQDs. The fluorescence images revealed labeling of the tumor endothelial cells by the contrast agent. Vessel wall-like structures outlined by labeled endothelium were clearly identified as indicated by the arrows. Labeling of blood vessels by RGD-pQDs was found as far as $1 \mathrm{~cm}$ outside the rim of the tumor, indicative of a widespread activation of vascular endothelium. No fluorescence was observed outside the blood vessels, which indicated that the RGD-QDs did not permeate the endothelium and target $\alpha \mathrm{v} \beta 3$-integrinexpressing tumor cells. Importantly, no endothelial cell labeling was found in blood vessels of distant normal tissues, e.g., muscle tissue of the hind limb and the skin of the ears (Fig. 2c; brightfield image and corresponding fluorescence image, respectively, of the same animal as in Fig. 2b). This observation demonstrated that the RGDQDs were specific for activated tumor endothelium only. As a control, pQDs that were not conjugated with RGDpeptides were injected. These QDs did not label endothelial cells, neither in the tumor (Fig. 2d) nor in the close proximity of the tumor (data not shown) and in the ear of the animal (Fig. 2f).

In vivo magnetic resonance imaging (MRI) was performed on animals $(n=4)$ that were anesthetized and kept
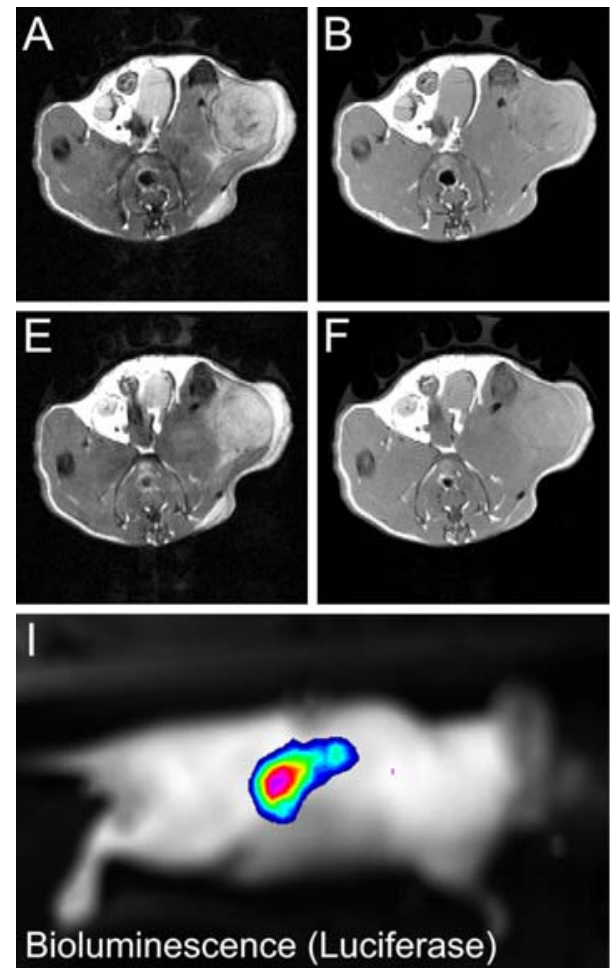

Fig. 3 Magnetic resonance and optical molecular imaging of tumor angiogenesis. a,h MR images of two slices through the tumor of a C57B16 mouse that was injected with RGD-pQDs. T2-weighted images (a,e), collected before the contrast agent was injected, show the contour of the tumor on the flank. T1-weighted images $(\mathbf{b}, \mathbf{c}, \mathbf{f}, \mathbf{g})$ with $\mathrm{TR}=800 \mathrm{~ms}$ were measured before $(\mathbf{b}, \mathbf{f})$ and $45 \mathrm{~min}$ after $(\mathbf{c}, \mathbf{g})$ the injection of the RGD-pQDs. The arrows in $(\mathbf{c}, \mathbf{g})$ indicate bright (positive contrast) regions in the periphery of the tumor. In $\mathbf{d}, \mathbf{h}$, pixels in the tumor with signal enhancement of at least three times the noise

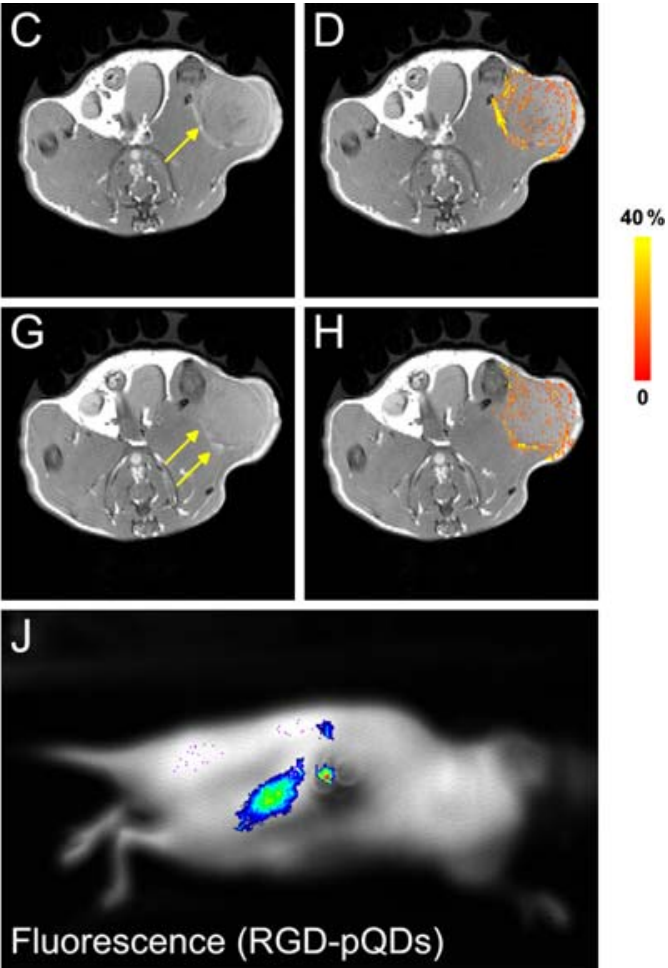

level are color coded according to the pseudo-color scale on the right. $\mathbf{i}, \mathbf{j}$ Bioluminescence imaging and fluorescence imaging of a Balb/c nude mouse with a luciferase-expressing renal carcinoma tumor after injection of luciferin (i). The strong bioluminescence signal is indicative of tumor growth in the right kidney. This signal colocalizes with a strong fluorescence signal (j) originating from intravenously administrated RGD-pQDs that are accumulated in the tumor 
warm using an in-house-built cradle. They were injected inside the MRI scanner by means of an infusion line placed in the tail vein to allow a direct and pixel-by-pixel comparison of the images pre- and post-contrast agent injection. First, the tumor was localized with T2-weighted imaging, in which the tumor appears as a hyperintense mass (Fig. 3a, e). High resolution T1-weighted images were acquired prior to and $45 \mathrm{~min}$ after contrast agent injection. The successful administration of the contrast agent was ascertained by monitoring the signal intensity increase in the aorta with the use of dynamic contrastenhanced T1-weighted imaging. In Fig. 3a-h, two slices through the tumor of a tumor-bearing mouse are depicted. Figure $3 b$ and $f$ show the T1-weighted MR images before contrast agent injection and Fig. $3 \mathrm{c}$ and g show images $45 \mathrm{~min}$ after contrast agent injection. Hyperintense regions were identified as indicated by the yellow arrows. In Fig. $3 \mathrm{~d}$ and $\mathrm{h}$, the pixel-by-pixel analysis of the images before and after injection of the contrast agent is presented for visualization of the statistically significant signal increase. The MR imaging voxels that showed significant signal enhancement following RGD-pQDs injection were mainly found at the tumor periphery, which corresponds with the regions of the tumor with highest angiogenic activity [17]. In previous studies, we applied RGD-conjugated paramagnetic liposomes to visualize angiogenesis in tumor-bearing mice with molecular MRI and observed a similar pattern of signal enhancement in peripheral regions of the tumor $[7,18]$.

Bioluminescence imaging of Balb/c nude mice that had been inoculated with luciferase-expressing renal carcinoma cells showed a high photon yield from the region of the kidney after i.p. injection of luciferin (Fig. 3i) that corresponds to the tumor. Fluorescence imaging was applied to these mice $10 \mathrm{~min}$ after intracardial injection of RGDpQDs. Strong fluorescent signal $\left(1,92 \mathrm{E}+10 \mathrm{Ph} / \mathrm{cm}^{2} / \mathrm{s}\right)$ was observed (Fig. 3j), which co-localized with the signal that originated from the luciferase expression site.

After the in vivo imaging experiments, animals were killed for fluorescence microscopy of QD localization in tumor and other tissues. Cell nuclei were stained with DAPI and appeared blue, while the QDs were visible in green. From the analysis of tumor tissues, it became evident that the RGD-pQDs showed a strikingly different distribution pattern from the non-targeted pQDs. In case of RGD-pQDs, a clear association with tumor endothelium was observed (Fig. 4a). Less fluorescence only in diffuse patterns was found in tumors of mice injected with pQDs (Fig. 4b). In muscle tissue, no RGD-pQDs or pQDs were found (Fig. 4c, d). Furthermore, the RGD-pQDs were observed to accumulate in the liver and spleen, while pQDs were not found accumulated in the liver and only moderately in the spleen (Fig. 4e-h). This bio-distribution pattern
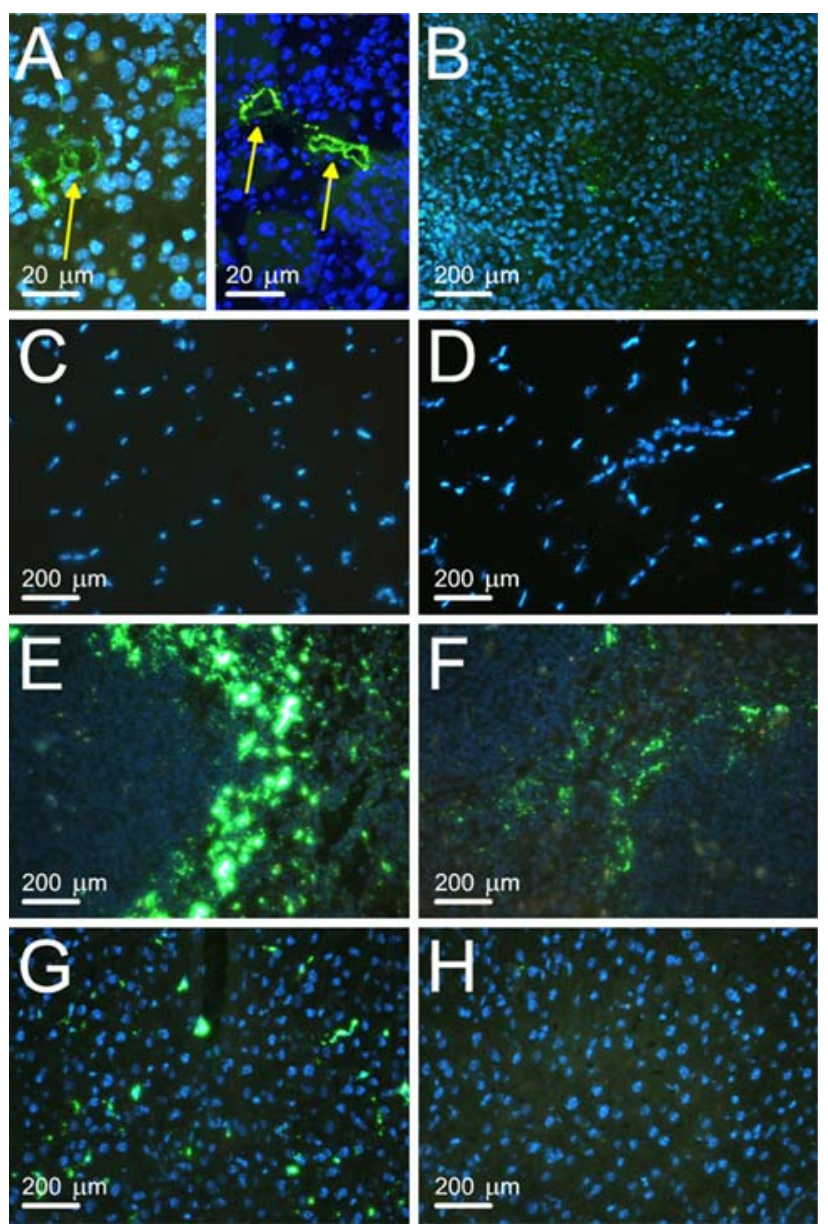

Fig. 4 Fluorescence microscopy of tissues from C57B16 mice that were killed 60 min after intravenously injecting RGD-pQDs or pQDs. Tissue sections $(10 \mu \mathrm{m})$ were counterstained with DAPI (blue) for visualization of nuclei. A clear association of RGD-pQDs (green) with the endothelium of the tumor vasculature was observed (a, yellow arrows). Bare pQDs did not home into tumor vasculature (b). A diffuse pattern of fluorescence within the tumor tissue was found for pQDs. Muscle tissue did not contain RGD-pQDs (c) or pQDs (d). RGD-pQDs accumulated in the spleen (e) and liver (g), while pQDs did accumulate in the spleen to a lesser extent (f) and were absent in the liver (h)

is in agreement with previous studies with other types of RGD conjugates [19].

\section{Discussion}

We have demonstrated that a novel paramagnetic quantum dot nanoparticle (RGD-pQDs) allows the visualization of tumor angiogenesis at the cellular level with IVM, at the macroscopic level with MRI and optical imaging. Intravital microscopy data showed widespread angiogenic activity mainly localized within the rim of the tumor and up to $1 \mathrm{~cm}$ from the tumor boundary. Furthermore, it was demonstrated that the RGD-pQDs associated with the activated 
endothelial cells rapidly after injection, which allowed identification of these cells within 5-10 min. In vivo MRI was demonstrated to be useful for the macroscopic localization of sites with high angiogenic activity, which again were mainly found at the rim of the tumor, while optical imaging was used to identify angiogenesis at the level of the whole animal. The present targeting findings are in agreement with what has been shown before with RGD conjugates. In case a nanoparticle or macromolecule is enriched with the $\alpha \mathrm{v} \beta 3$-specific RGD-peptide developed by Kok et al. [15], the probe targets the tumor endothelial cells and does not or marginally extravasate. In 2002, a pharmacokinetic and cellular distribution study was performed with RGD-modified proteins that extensively describe this phenomenon [19]. Furthermore, liposomes conjugated with the same RGD-peptide have been described to target tumor endothelial cells without extravasating in several drug targeting [20, 21] and molecular imaging studies [7, 18]. Other nanoparticles equipped with $\alpha \mathrm{v} \beta 3$ antagonists were also found to target the angiogenic endothelium without extravasation $[22,23]$. The reason that such conjugates do not extravasate from the circulation and merely bind to activated endothelial cells is twofold. The size of the conjugates is of importance and permeability becomes increasingly restrictive with increasing size. In addition and perhaps more importantly, multiple targeting moieties located on one scaffold enhance the affinity of the conjugate for the target. This phenomenon is known as multivalency and likely plays an important role in the high affinity RGD-pQDs have for tumor blood vessels [19].

Although this study convincingly demonstrates the use of RGD-pQDs for multimodality imaging of tumor angiogenesis, two limitations of this study need to be addressed. Firstly, the nature of the micelle-coated QDs is such that they can be excellently visualized in vivo using IVM. On the other hand, the visualization of the QDs in frozen sections is possible as shown in Fig. 4, but ex vivo immunohistochemical co-staining for different markers remains difficult because these procedures solubilize the QD coating, which subsequently leads to loss of fluorescence. Secondly, direct immunohistochemical staining of $\alpha v \beta 3$-integrin expression remains difficult because of the absence of a suitable antibody specific for mice. In other species, like the rabbit, several research groups have shown the application of the $\alpha \mathrm{v} \beta 3$-specific antibody LM609 for this purpose $[4,6,24]$. In mice targeting studies, the majority of the publications does not address immunohistochemical detection of $\alpha \mathrm{v} \beta 3$-expression $[5,15,19-21$, $25]$. Alternatively, the $\alpha \mathrm{v}$ - and $\beta 3$-subunits can be identified independently, which would, together with an endothelial marker staining, give some information about $\alpha \mathrm{v} \beta 3$-expression on endothelial cells. Still, this triple staining method remains difficult and because of the expression of other integrins that contain either the $\alpha \mathrm{v}$ - or $\beta 3$-subunit this strategy may lead to an erroneous interpretation of the actual $\alpha \mathrm{v} \beta 3$-expression. Angiostatic therapy based on RGD-peptides on the mouse models used in our study have shown to reduce tumor growth and vascularization. Together with the abovementioned studies that have employed the specific RGD-peptide used in the current study before it can be concluded that $\alpha \mathrm{v} \beta 3$ expression on the tumor vasculature in these mouse models is abundant and can be exploited for targeting purposes.

In conclusion, we are the first to have shown the unique possibilities of a paramagnetic quantum dot preparation for parallel in vivo optical and MR imaging studies. The nanotechnology presented here opens new and exciting opportunities for the molecular imaging assessment of many pathophysiological processes in animal models.

Acknowledgments This study was funded in part by the EC-FP6project DiMI, LSHB-CT-2005-512146 - and by the BSIK program entitled Molecular Imaging of Ischemic Heart Disease (project number BSIK03033). The authors thank Dr. Jeff Bulte from the Johns Hopkins University School of Medicine for critically reading the manuscript, and Drs. Celso de Mello Donegá and Rolf Koole from the Utrecht University for useful discussions.

Open Access This article is distributed under the terms of the Creative Commons Attribution Noncommercial License which permits any noncommercial use, distribution, and reproduction in any medium, provided the original author(s) and source are credited.

\section{References}

1. McDonald DM, Choyke PL (2003) Imaging of angiogenesis: from microscope to clinic. Nat Med 9:713-725. doi:10.1038/ nm0603-713

2. Griffioen AW, Molema G (2000) Angiogenesis: potentials for pharmacologic intervention in the treatment of cancer, cardiovascular diseases, and chronic inflammation. Pharmacol Rev $52: 237-268$

3. Costouros NG, Diehn FE, Libutti SK (2002) Molecular imaging of tumor angiogenesis. J Cell Biochem Suppl 39:72-78. doi: $10.1002 /$ jcb. 10426

4. Sipkins DA, Cheresh DA, Kazemi MR, Nevin LM, Bednarski MD, Li KC (1998) Detection of tumor angiogenesis in vivo by alphaVbeta3-targeted magnetic resonance imaging. Nat Med 4:623-626. doi:10.1038/nm0598-623

5. Haubner R, Wester HJ, Weber WA, Mang C, Ziegler SI, Goodman SL, Senekowitsch-Schmidtke R, Kessler H, Schwaiger M (2001) Noninvasive imaging of alpha(v)beta3 integrin expression using 18F-labeled RGD-containing glycopeptide and positron emission tomography. Cancer Res 61:1781-1785

6. Winter PM, Caruthers SD, Kassner A, Harris TD, Chinen LK, Allen JS, Lacy EK, Zhang H, Robertson JD, Wickline SA, Lanza GM (2003) Molecular imaging of angiogenesis in nascent Vx-2 rabbit tumors using a novel alpha(nu)beta3-targeted nanoparticle and 1.5 tesla magnetic resonance imaging. Cancer Res 63:5838-5843

7. Mulder WJ, Strijkers GJ, Habets JW, Bleeker EJ, van der Schaft DW, Storm G, Koning GA, Griffioen AW, Nicolay K (2005) MR molecular imaging and fluorescence microscopy for identification 
of activated tumor endothelium using a bimodal lipidic nanoparticle. FASEB J 19:2008-2010

8. Veiseh O, Sun C, Gunn J, Kohler N, Gabikian P, Lee D, Bhattarai N, Ellenbogen R, Sze R, Hallahan A, Olson J, Zhang MQ (2005) Optical and MRI multifunctional nanoprobe for targeting gliomas. Nano Lett 5:1003-1008. doi:10.1021/n10502569

9. Persigehl T, Heindel W, Bremer C (2005) MR and optical approaches to molecular imaging. Abdom Imaging 30:342-354. doi:10.1007/s00261-004-0230-3

10. Michalet X, Pinaud FF, Bentolila LA, Tsay JM, Doose S, Li JJ, Sundaresan G, Wu AM, Gambhir SS, Weiss S (2005) Quantum dots for live cells, in vivo imaging, and diagnostics. Science 307:538-544. doi:10.1126/science.1104274

11. Medintz IL, Uyeda HT, Goldman ER, Mattoussi H (2005) Quantum dot bioconjugates for imaging, labelling and sensing. Nat Mater 4:435-446. doi:10.1038/nmat1390

12. Gao X, Cui Y, Levenson RM, Chung LW, Nie S (2004) In vivo cancer targeting and imaging with semiconductor quantum dots. Nat Biotechnol 22:969-976. doi:10.1038/nbt994

13. Stroh M, Zimmer JP, Duda DG, Levchenko TS, Cohen KS, Brown EB, Scadden DT, Torchilin VP, Bawendi MG, Fukumura D, Jain RK (2005) Quantum dots spectrally distinguish multiple species within the tumor milieu in vivo. Nat Med 11:678-682. doi: $10.1038 / \mathrm{nm} 1247$

14. Mulder WJ, Koole R, Brandwijk RJ, Storm G, Chin PT, Strijkers GJ, de Mello DC, Nicolay K, Griffioen AW (2006) Quantum dots with a paramagnetic coating as a bimodal molecular imaging probe. Nano Lett 6:1-6. doi:10.1021/n1051935m

15. Kok RJ, Schraa AJ, Bos EJ, Moorlag HE, Asgeirsdottir SA, Everts M, Meijer DK, Molema G (2002) Preparation and functional evaluation of RGD-modified proteins as alpha(v)beta(3) integrin directed therapeutics. Bioconjug Chem 13:128-135. doi: $10.1021 / \mathrm{bc} 015561+$

16. van der Schaft DW, Dings RP, de Lussanet QG, van Eijk LI, Nap AW, Beets-Tan RG, Bouma-Ter Steege JC, Wagstaff J, Mayo KH, Griffioen AW (2002) The designer anti-angiogenic peptide anginex targets tumor endothelial cells and inhibits tumor growth in animal models. FASEB J 16:1991-1993

17. de Lussanet QG, Beets-Tan RG, Backes WH, van der Schaft DW, van Engelshoven JM, Mayo KH, Griffioen AW (2004) Dynamic contrast-enhanced magnetic resonance imaging at 1.5 Tesla with gadopentetate dimeglumine to assess the angiostatic effects of anginex in mice. Eur J Cancer 40:1262-1268. doi:10.1016/j.ejca. 2004.01.020

18. Mulder WJ, van der Schaft DW, Hautvast PA, Strijkers GJ, Koning GA, Storm G, Mayo KH, Griffioen AW, Nicolay K (2007) Early in vivo assessment of angiostatic therapy efficacy by molecular MRI. FASEB J 21:378-383. doi:10.1096/fj.066791com

19. Schraa AJ, Kok RJ, Moorlag HE, Bos EJ, Proost JH, Meijer DK, de Leij LF, Molema G (2002) Targeting of RGD-modified proteins to tumor vasculature: a pharmacokinetic and cellular distribution study. Int J Cancer 102:469-475. doi:10.1002/ijc. 10727

20. Janssen AP, Schiffelers RM, ten Hagen TL, Koning GA, Schraa AJ, Kok RJ, Storm G, Molema G (2003) Peptide-targeted PEGliposomes in anti-angiogenic therapy. Int $\mathrm{J}$ Pharm 254:55-58. doi:10.1016/S0378-5173(02)00682-8

21. Schiffelers RM, Koning GA, ten Hagen TL, Fens MH, Schraa AJ, Janssen AP, Kok RJ, Molema G, Storm G (2003) Anti-tumor efficacy of tumor vasculature-targeted liposomal doxorubicin. J Control Release 91:115-122. doi:10.1016/S0168-3659(03) 00240-2

22. Hu G, Lijowski M, Zhang H, Partlow KC, Caruthers SD, Kiefer G, Gulyas G, Athey P, Scott MJ, Wickline SA, Lanza GM (2007) Imaging of $\mathrm{Vx}-2$ rabbit tumors with alpha(nu)beta3-integrin-targeted 111In nanoparticles. Int J Cancer 120:1951-1957. doi: 10.1002/ijc. 22581

23. Smith BR, Cheng Z, De A, Koh AL, Sinclair R, Gambhir SS (2008) Real-time intravital imaging of RGD-quantum dot binding to luminal endothelium in mouse tumor neovasculature. Nano Lett 8(9):2599-2606

24. Winter PM, Morawski AM, Caruthers SD, Fuhrhop RW, Zhang H, Williams TA, Allen JS, Lacy EK, Robertson JD, Lanza GM, Wickline SA (2003) Molecular imaging of angiogenesis in earlystage atherosclerosis with alpha(v)beta3-integrin-targeted nanoparticles. Circulation 108:2270-2274. doi:10.1161/01.CIR.00000 93185.16083.95

25. Schmieder AH, Winter PM, Caruthers SD, Harris TD, Williams TA, Allen JS, Lacy EK, Zhang H, Scott MJ, Hu G, Robertson JD, Wickline SA, Lanza GM (2005) Molecular MR imaging of melanoma angiogenesis with alphanubeta3-targeted paramagnetic nanoparticles. Magn Reson Med 53:621-627. doi:10.1002/ mrm.20391 